\title{
La mortalidad infantil por malformaciones congénitas en México: un problema de oportunidad y acceso al tratamiento
}

\author{
Alejandro V. Gómez-Alcalá1 y Ramón A. Rascón-Pacheco²
}

Forma de citar Gómez-Alcalá AV, Rascón-Pacheco RA. La mortalidad infantil por malformaciones congénitas en México: un problema de oportunidad y acceso al tratamiento. Rev Panam Salud Publica. 2008;24(5): 297-303.

RESUMEN Objetivos. Caracterizar el comportamiento del Sistema Nacional de Salud (SNS) de México en la tarea de reducir el número de muertes por malformaciones congénitas mediante el análisis de la tendencia de las tasas de mortalidad infantil específicas por esas afecciones.

Métodos. Análisis de series de tiempos de las defunciones de niños y niñas menores de 1 año entre 1980 y 2005, según las bases de datos nacionales anuales de mortalidad de la Secretaría de Salud de México. Se calcularon las tasas de mortalidad infantil específicas (TMIe) por malformaciones congénitas graves más frecuentes en México: defectos del tubo neural, hernia diafragmática congénita, exonfalos (onfalocele y gastrosquisis) y malformaciones cardíacas y del tubo digestivo, agrupadas según el grado de urgencia y de sofisticación tecnológica que demanda su tratamiento y el desenlace más frecuente.

Resultados. Entre 1980 y 2005, la tasa de mortalidad infantil en México descendió de $40,7$ a 16,9 por 1000 nacimientos ( $\beta=-0,86 ; \mathrm{P}<0,001)$; en cambio, la tasa de mortalidad específica por malformaciones congénitas creció de 2,2 a 3,5 por 1000 nacimientos $(\beta=0,05$; $\mathrm{P}<0,001)$. La hipertrofia pilórica y la atresia anorrectal, malformaciones con buen pronóstico y tratamiento programable en unidades con equipamiento básico, fueron las únicas que mostraron una tendencia descendente en su TMIe $(\beta=-0,01$ a $-0,09 ; \mathrm{P}<0,001)$, mientras que las que requieren tratamiento urgente en unidades especializadas mostraron TMIe crecientes $(\beta=0,03$ a 0,$05 ; \mathrm{P}<0,001)$.

Conclusiones. El desarrollo del SNS de México entre 1980 y 2005 no se ha traducido en una reducción en la mortalidad por malformaciones congénitas; esta ineficacia fue más notoria en las enfermedades cuyo tratamiento es urgente y requiere tecnología sofisticada.

Palabras clave Mortalidad infantil, tasa de mortalidad infantil, anomalías congénitas, México.

\footnotetext{
Instituto Mexicano del Seguro Social, Delegación Sonora, Coordinación de Investigación en Salud, Ciudad Obregón, Sonora, México. La correspondencia se debe dirigir a Alejandro Gómez Alcalá, Instituto Mexicano del Seguro Social, Delegación Sonora, Coordinación de Investigación en Salud, Av. 5 de Febrero No. 220 norte, Colonia Centro, Ciudad Obregón, Sonora 85000, México. Correo electrónico: alejandro.gomezal@imss.gob.mx

2 Instituto Mexicano del Seguro Social, Unidad de Investigación Epidemiológica y en Servicios de Salud, Hermosillo, Sonora, México.
}

En los últimos 50 años, la tasa de mortalidad infantil (TMI) ha disminuido en gran parte del mundo gracias a la adopción de diversas medidas en las áreas de la salud pública, la medicina preventiva y la atención médica (1). No obstante, esa disminución no es homogénea: si bien la mortalidad actual atribuible a las infecciones intestinales y pulmonares es mucho menor hoy que hace una década, la asociada con los partos prematuros y las malformaciones congénitas (MC) ha aumentado (2).

Las mayores tasas de mortalidad y morbilidad asociadas con las MC afectan negativamente a los sistemas de salud y son causa de gran preocupación, no solo por los recursos que consumen (3), sino también por las afec- 
FIGURA 1. Recursos para la salud y tasas de mortalidad infantil, México, 1980-2005

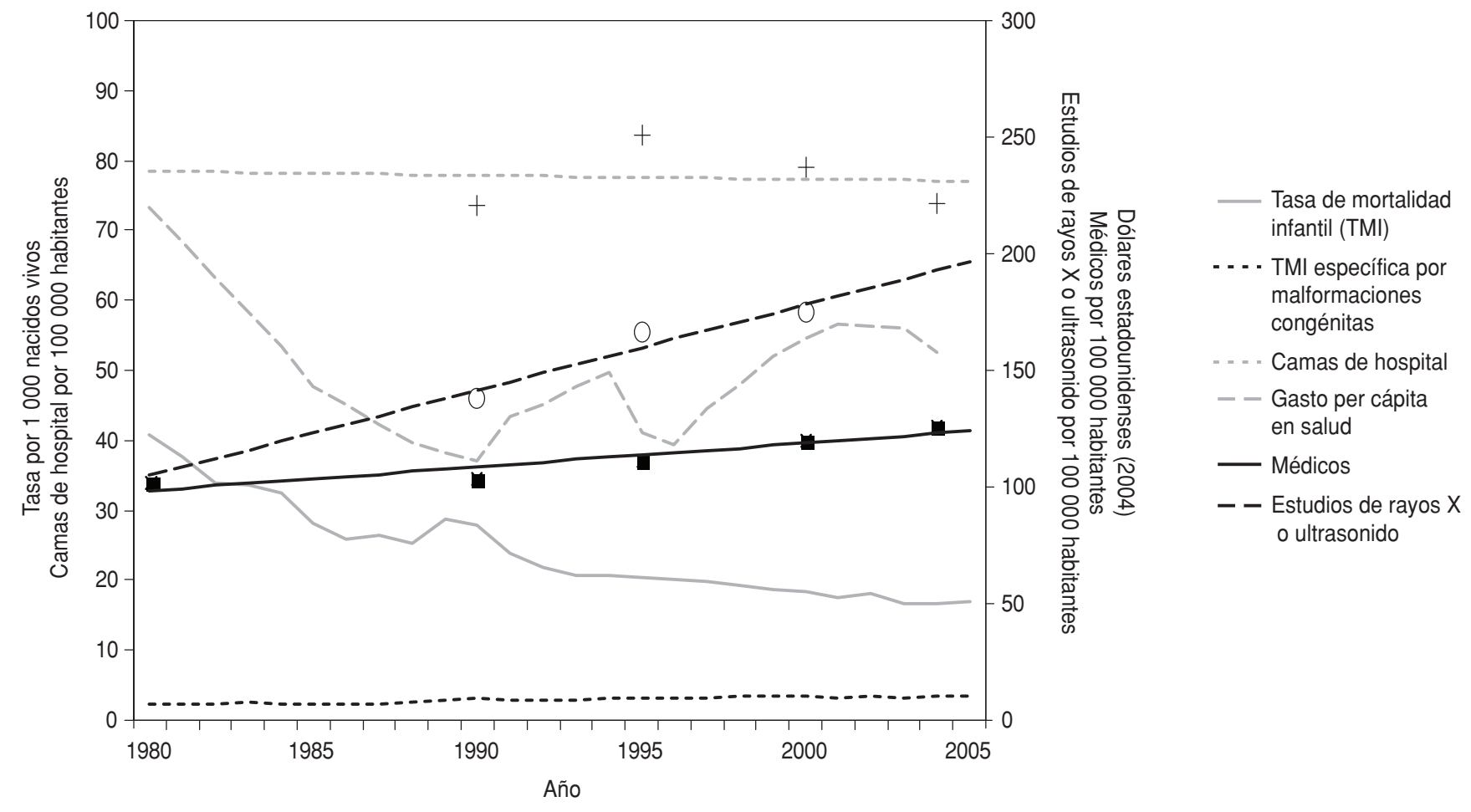

taciones sociales que ocasionan, en especial a la madre y a las personas cercanas a ella. Las lagunas ontológicas existentes con relación a los derechos de los embriones y al lugar que ocupan en la sociedad han llevado a que se mantenga viva la discusión sobre las implicaciones éticas y legales relacionadas con la decisión de interrumpir el embarazo cuando el feto presenta malformaciones confirmadas graves (4).

En amplios sectores de la sociedad mexicana prevalece una visión limitada sobre las MC que se centra solamente en el período posnatal y no presta la atención necesaria a las acciones preventivas y paliativas del daño que se pueden operar durante la gestación. Esta óptica ha impedido asumir una estrategia formal frente al desafío que representan las $\mathrm{MC}$, pues el único enfoque terapéutico avalado por el Sistema Nacional de Salud (SNS) mexicano es el reconstructivo-curativo, basado en una red de hospitales y servicios de primer y segundo niveles con acceso restringido al tercer nivel, muchos de ellos inaccesibles para buena parte de la población. La aprobación de leyes que brinden protección a las familias afectadas y permitan la interrupción de embarazos por MC fetales graves ha ocurrido en menos de la mitad de los estados mexicanos, en tanto que tan sólo en el Distrito Federal se han abierto unidades de atención médica para la mujer embarazada que opte por el aborto; esto lleva muchas veces a recurrir a procedimientos riesgosos realizados al margen de la ley, las instituciones públicas de salud y los sistemas de registro.

Entre 1980 y 2005 en México se introdujeron los importantes avances verificados en la tecnología de imágenes por ultrasonido y los tratamientos quirúrgicos pediátricos y se situaron especialistas en la mayoría de los centros urbanos. En ese lapso, la población mexicana aumentó de 62 a 104 millones de habitantes y el SNS tuvo que ampliar considerablemente su cobertura para satisfacer las necesidades crecientes (figura 1). Sin embargo, estas intenciones tropezaron con dos graves crisis financieras que sacudieron México (de 1982 a 1987 y de 1995 a
1999) e impusieron restricciones en el gasto público que llevaron a recortar a la mitad la inversión total en salud (5, 6). Como resultado de ese proceso, los presupuestos se ajustaron a las prioridades de la salud pública, en detrimento del gasto asistencial y curativo. Esto llevó al desequilibrio del SNS, a un retraso en la incorporación de los avances médicos y tecnológicos relacionados con la asistencia médica y a un retroceso en la eficacia de la atención de pacientes con insuficiencias orgánicas graves. Es posible que la calidad de la atención de los infantes con MC haya sentido también el efecto negativo de estos ajustes económicos.

Si bien el diverso grado de complejidad de las MC abre un espectro muy heterogéneo de retos para los sistemas de salud, el acceso de los niños a tratamientos resolutivos debe ser equitativo, tanto para los pacientes con malformaciones sencillas como para los que presentan anomalías que demandan tratamientos quirúrgicos urgentes o el uso de tecnologías más sofisticadas. El aumento en la mortalidad por $\mathrm{MC}$ en niños y niñas menores de 1 año 
que presentan trastornos cuyo tratamiento reviste una mayor urgencia o requiere de unidades especializadas (UE) que cuenten con tecnologías más sofisticadas puede ser un reflejo de la falta de equidad en el SNS mexicano en detrimento de estos pacientes.

Por todo esto se hace necesario evaluar la eficacia del enfoque asistencial vigente en México en la reducción del número de muertes infantiles por $\mathrm{MC}$, así como el desempeño general del sistema de salud ante los casos más graves. En el presente trabajo se analiza la tendencia de las tasas de mortalidad infantil específicas (TMIe) por MC, con el objetivo de caracterizar el comportamiento del SNS de México en la tarea de reducir el número de muertes por esa causa.

\section{MATERIALES Y MÉTODOS}

Se realizó un análisis de series de tiempo a partir de los datos contenidos en las bases de datos nacionales anuales de mortalidad de la Secretaría de Salud de México. Se analizaron las defunciones en menores de 1 año ocurridas entre 1980 y 2005, según el capítulo XIV (anomalías congénitas) de la Clasificación Estadística Internacional de Enfermedades, Traumatismos y Causas de Defunción, 9. ${ }^{\text {a }}$ revisión (CIE9), para los datos del período 1980 a
1998 (7) y el capítulo XVII (malformaciones congénitas, deformidades y anomalías cromosómicas) de la Clasificación Estadística Internacional de Enfermedades y Problemas Relacionados con la Salud, 10. a edición (CIE-10), para los datos de 1999 a 2005 (8).

Se tomaron en cuenta las MC graves más frecuentes en México: defectos del tubo neural, hernia diafragmática congénita, exónfalos (onfalocele y gastrosquisis) y malformaciones cardíacas y del tubo digestivo. Como un primer paso, se crearon categorías de enfermedades según su presentación e impacto clínico (cuadro 1) y posteriormente se agruparon de acuerdo con el grado de urgencia y de sofisticación tecnológica que demanda su tratamiento y el desenlace más frecuente, de la siguiente forma:

Grupo 1: MC intratables y de letalidad absoluta (anencefalia)

Grupo 2: MC de tratamiento urgente en UE con equipamiento sofisticado y con pronóstico incierto (hernia diafragmática congénita y malformaciones valvulares de vías de salida y conexiones venosas del corazón)

Grupo 3: MC de tratamiento urgente en UE con equipamiento sofisticado y con pronóstico favorable (atresia esofágica, atresia intestinal y exonfalos)

Grupo 4: MC de tratamiento no urgente en UE con equipamiento sofis-

CUADRO 1. Malformaciones congénitas analizadas según su categoría

\begin{tabular}{lcc}
\hline \multicolumn{1}{c}{ Malformación } & Código ClEga & Código CIE10 \\
\hline Anencefalia & 740 & Q00,0 \\
Espina bífida & 741 & Q05 \\
Malformaciones congénitas de las cámaras cardíacas, & & \\
$\quad$ sus conexiones y los tabiques cardíacos & 745 & Q20-Q21 \\
Malformaciones de las válvulas pulmonar y tricúspide & $746,0-746,2$ & Q22 \\
Estenosis del infundíbulo pulmonar & 746,83 & Q24,3 \\
Corazón izquierdo hipoplásico & 746,7 & Q23,4 \\
Conexión anómala de venas pulmonares & 747,4 & Q26,2-Q26,4 \\
Malformaciones congénitas del esófago & $750,3-750,4$ & Q39 \\
Estenosis hipertrófica pilórica congénita & 750,5 & Q40,0 \\
Atresia y estenosis del intestino delgado & 751,1 & Q41 \\
Atresia y estenosis del intestino grueso, recto y canal anal & 751,2 & Q42 \\
Hernia diafragmática congénita & 756,6 & Q79,0 \\
Agenesia diafragmática & & Q79,1 \\
Anomalías de la pared abdominal: gastrosquisis y onfalocele & 756,7 & Q79,2-Q79,3 \\
Anomalías congénitas (global) & $740-759$ & Q00-Q99 \\
\hline
\end{tabular}

\footnotetext{
a Según el Manual de clasificación estadística internacional de enfermedades, traumatismos y causas de defunción (7).
}

b Según la Clasificación estadística internacional de enfermedades y problemas relacionados con la salud. 10. ${ }^{a}$ revisión (8). ticado y con pronóstico favorable (malformaciones del bulbo arterioso y del cierre del tabique cardíaco)

Grupo 5: MC de posible tratamiento en hospitales generales, sin urgencia, y con pronóstico bueno (hipertrofia pilórica, atresia anorrectal y espina bífida).

Para el cálculo de las tasas de mortalidad general y específicas se utilizó la cohorte por 1000 nacimientos anuales como denominador; esta cifra se tomó de las estimaciones del Consejo Nacional de Población (9).

La descripción de los cambios observados en las tasas de mortalidad (tendencias) se hizo mediante un modelo de regresión lineal simple con el programa informático Stata v. 9,0.

\section{RESULTADOS}

Durante los 26 años estudiados, a pesar de los recortes del gasto en salud operados como resultado de las crisis económicas y sus efectos sobre la estructura y funcionamiento del SNS, la TMI en México decreció de 40,7 a 16,9 por 1000 nacimientos $(\beta=-0,86$; $P<0,0001)$. En cambio, en el mismo lapso, la TMIe por MC en México creció de 2,2 a 3,5 por 1000 nacimientos $(\beta=0,05 ; P<0,0001)$ (figura 1). La proporción nacional de muertes infantiles atribuibles a MC creció de 5,5\% a $21,0 \%$.

En el grupo 1, no se observaron cambios significativos en la tendencia de la TMIe por anencefalia $(\beta=-0,0002$, $P=0,9)$, aunque llama la atención la alta meseta observada entre 1988 y 1999 (figura 2). El valor de la TMIe por esta MC en 2005 fue de 0,17 por 1000 nacimientos.

En el grupo 2, las mortalidades por malformaciones valvulares y por hernia diafragmática congénita mostraron una ligera pero significativa tendencia a aumentar $(\beta=0,03 ; P<0,0001$ en ambas), con valores de las TMIe en 2005 de 0,09 y 0,07 por 1000 nacimientos, respectivamente (figura 3). Esto puede deberse a fallas en la aplicación de los tratamientos oportunos en las unidades especializadas. 
En el grupo 3, los valores de mortalidad por atresia esofágica $(\beta=0,001$; $P=0,5)$ y atresia intestinal $(\beta=0,0007$; $P=0,8$ ) no mostraron una tendencia creciente estadísticamente significativa en los 25 años analizados; el valor de la TMIe en el año 2005 fue de 0,05 por 1000 nacimientos en ambos casos. Con respecto a los casos de exonfalos, se observó un aumento significativo en la mortalidad ( $\beta=0,05 ; P<0,0001)$, con valores de las TMIe en 2005 de 0,11 por 1000 nacimientos (figura 4). Esto también puede ser el reflejo de fallas en la aplicación de los tratamientos oportunos en las unidades especializadas.

En el grupo 4, la mortalidad por malformaciones de las cámaras y tabiques cardíacos mostró una significativa tendencia a aumentar $(\beta=0,06 ; P<0,0001)$ y alcanzó en 2005 una TMle de 0,23 por 1000 nacimientos (figura 2). Mientras, en el grupo 5, la mortalidad por espina bífida presentó una tendencia a aumentar hasta 1989, con una TMIe máxima de 0,35 por 1000 nacimientos, para después mostrar un declive hasta un valor de la TMIe en 2005 de 0,05 por 1000 nacimientos $(\beta=-0,09 ; P<0,0001)$. La mortalidad por hipertrofia pilórica ( $\beta=$ $-0,013 ; P<0,001)$ y atresia anorrectal ( $\beta=-0,072 ; P<0,001)$ presentaron una tendencia decreciente uniforme a lo largo del período estudiado, con una TMIe para 2005 de 0,01 en ambos casos (figura 5); al parecer, la aplicación de tratamiento para estas enfermedades en hospitales de segundo nivel de atención ha resultado eficaz.

\section{DISCUSIÓN}

La tendencia decreciente en la TMI es un indicador de eficacia de los sistemas de salud, independientemente de la constitución étnica de la población (10). Sin embargo, se han encontrado grandes variaciones en las tendencias de las TMIe por MC entre países de diferentes zonas geoeconómicas (11).

El presente estudio corrobora, por un lado, el efecto negativo creciente de las MC sobre la mortalidad infantil en México, no solo en términos proporcionales — desde 1995 son la segunda causa de muerte en esta categoría-
FIGURA 2. Tendencia de la tasa de mortalidad infantil específica por anencefalia y malformaciones de la cámara y los tabiques cardíacos, México, 1980-2005

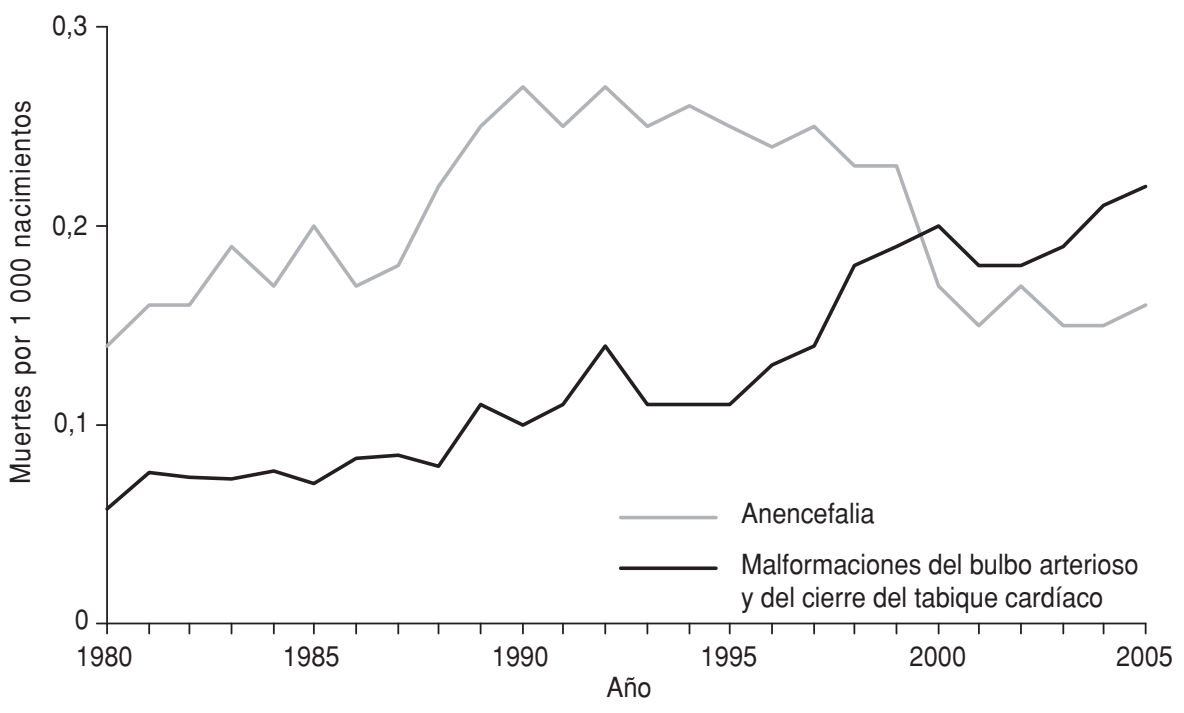

FIGURA 3. Tendencia de la tasa de mortalidad infantil específica por malformaciones cardíacas y diafragmáticas graves, México, 1980-2005

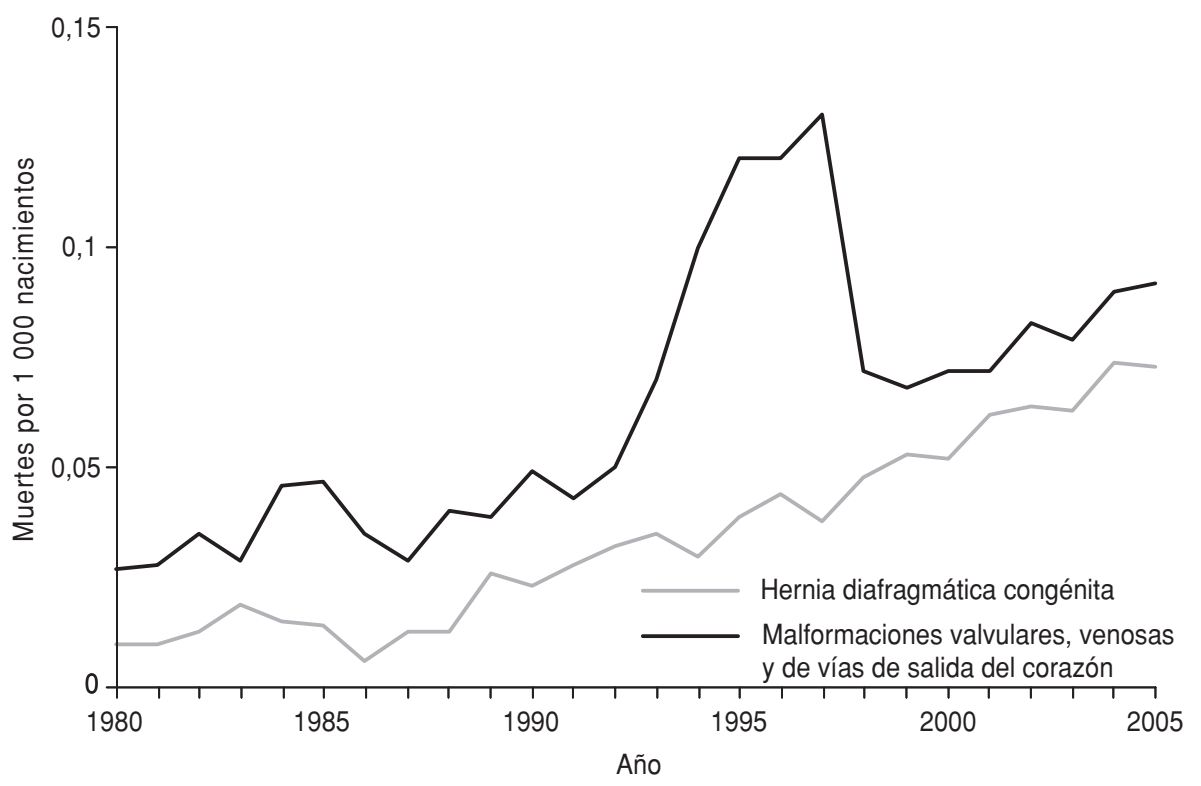

sino absolutos, con un aumento en su TMIe que contrasta con el descenso observado en la TMI. La heterogeneidad observada en las tendencias de las TMIe por las diferentes MC estudiadas se podría explicar por las diferencias en la oferta asistencial que el SNS brinda a estos infantes, probablemente debido a la orientación preferencial del gasto en salud hacia la atención médica de menor especialización.
Las TMIe por MC que no requieren tratamiento de urgencia o el empleo de tecnologías sofisticadas - como la hipertrofia pilórica y la atresia anorrectal- fueron las únicas identificadas en este estudio con tendencia descendente. Esta disminución de las TMIe no debe atribuirse a la disminución de su incidencia $(12,13)$, sino al aumento en la eficacia del sistema de salud, al brindar tratamientos relativa- 
FIGURA 4. Tendencia de la tasa de mortalidad infantil específica por malformaciones graves del tubo digestivo y de la pared abdominal, México, 1980-2005

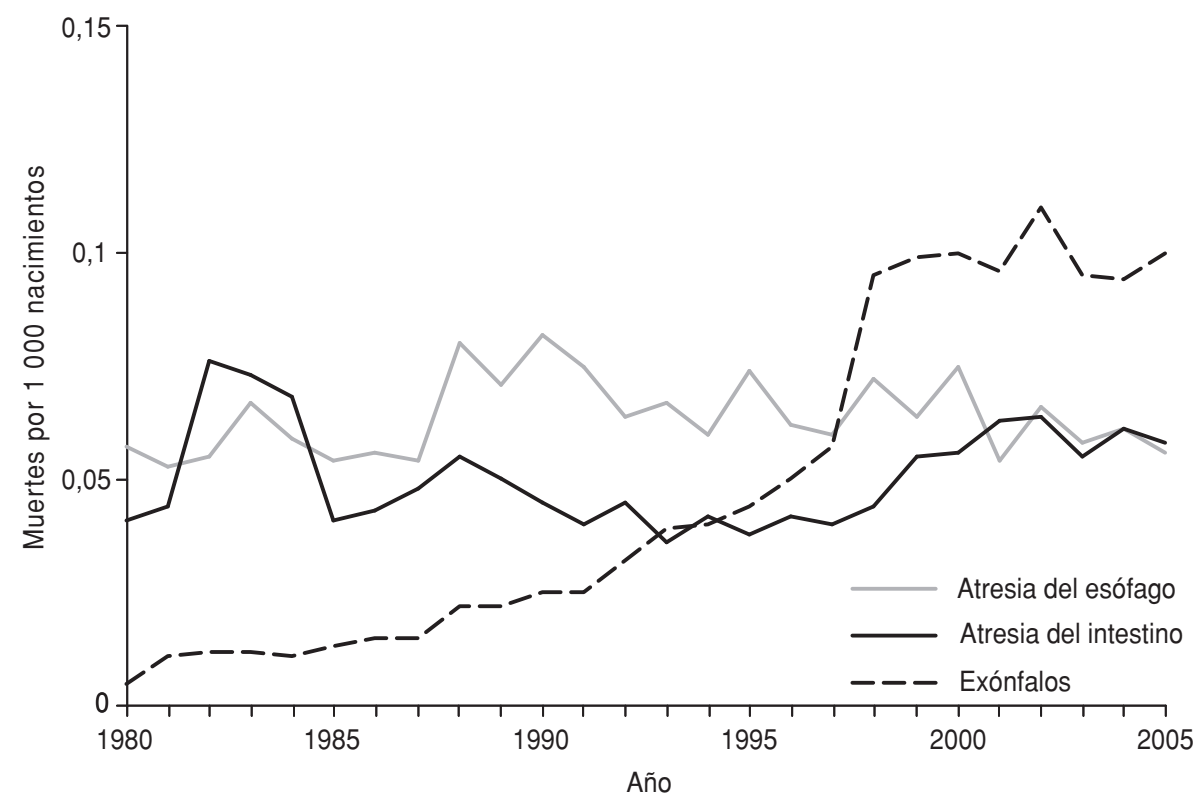

FIGURA 5. Tendencia de la tasa de mortalidad infantil específica por malformaciones del tubo digestivo y del tubo neural, México, 1980-2005

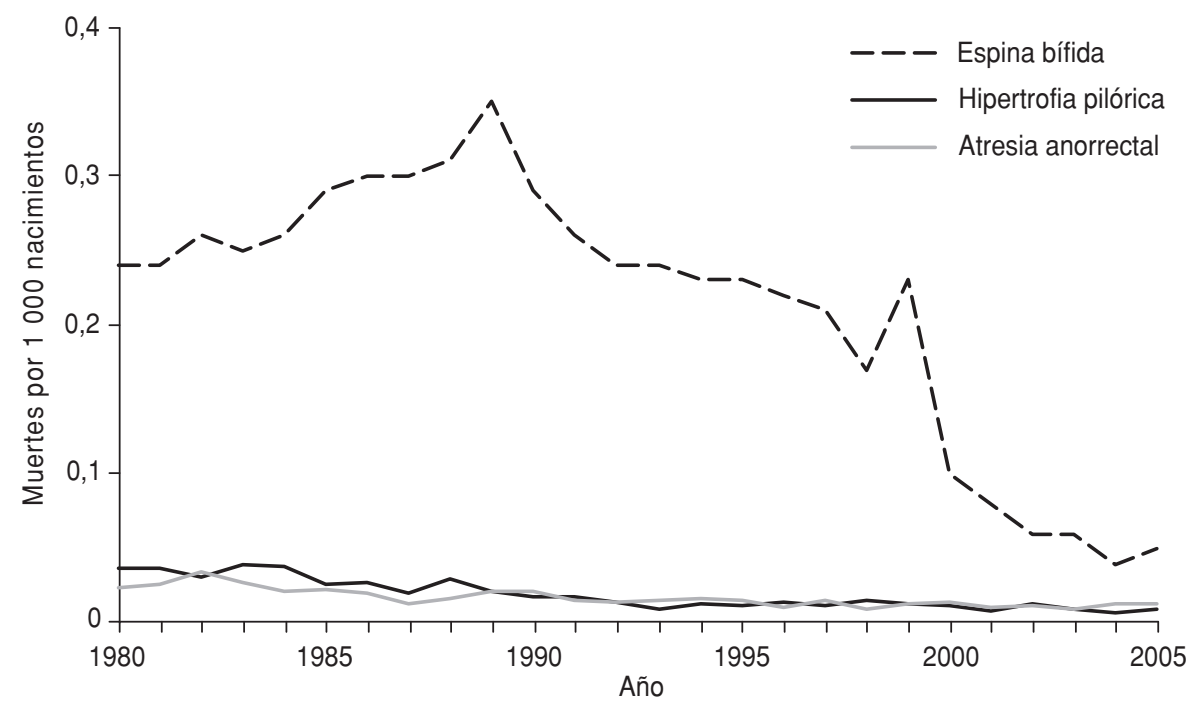

mente sencillos en hospitales generales de segundo nivel.

Algo muy distinto ocurre con las TMIe por MC que requieren el empleo de tecnologías sofisticadas en UE, en las que no se observan reducciones, a pesar del incremento en el número de especialistas y los avances terapéuticos registrados en los últimos años. En estos casos, el SNS de México demuestra un rezago mayor que los sistemas de salud de otros países (14-16), lo que también otros factores, como una mayor cobertura y exactitud del sistema de registro de muertes en los últimos años o un aumento en la incidencia de estos padecimientos. Es importante, además, destacar que el diagnóstico de algunas de las MC analizadas depende de que las unidades médicas cuenten con los especialistas necesarios y el equipamiento y los instrumentos adecuados. El mejoramiento en los recursos humanos y el equipamiento verificado de manera paulatina a lo largo de las últimas décadas puede explicar parcialmente la tendencia ascendente en el número de casos diagnosticados con malformaciones cardíacas y hernia diafragmática (17).

Esta mayor capacidad de diagnóstico debe haber permitido identificar más casos de estas enfermedades $\mathrm{y}$, paradójicamente, puede haber contribuido al incremento observado en la tasa de mortalidad por MC. No obstante, lejos de constituir un testimonio del progreso del SNS de México, esta paradoja puede estar evidenciando un desequilibrio en su desarrollo por haber desatendido la capacidad asistencial reconstructiva. De haber crecido esta a la par de la capacidad diagnóstica, se habría observado un aumento en la supervivencia de los infantes con MC graves y se habría detenido - $\mathrm{O}$ incluso invertido - la tendencia creciente de las TMIe por MC graves. Así ha ocurrido en los países con mayor renta per cápita $(18,19)$, pero no en los de América Latina y de Europa Oriental (20), lo que sugiere que este renglón de la atención infantil es sensible a la magnitud de la inversión en salud.

Las dificultades al interpretar las tendencias de la TMIe se pueden apreciar en el caso de las MC del tubo neural, cuyo pico entre 1985 y 1990 no se podría explicar solamente por las mejoras en los sistemas de registro de enfermedades (21) y cuyo descenso a partir de 1997 no obedece a una mayor eficacia terapéutica. Este comportamiento podría deberse a la reducción en la incidencia gracias al programa de fortificación de los alimentos con folatos (22), establecido en México en 1999, o al aumento en el número de interrupciones de embarazos con estos 
trastornos, aunque este último procedimiento solo está al alcance de quienes tienen acceso a la confidencialidad de la medicina privada.

La ineficacia del SNS mexicano para reducir la mortalidad por las MC que requieren tratamientos de urgencia y el empleo de tecnologías sofisticadas puede deberse a su insuficiente estructura y mala organización - como lo evidencia la falta de un sistema nacional de vigilancia epidemiológica de MC - y a la escasa inversión asistencial en los hospitales (23). Las llamadas décadas perdidas (de 1980 a 2000), con la consiguiente reducción en el gasto social, y la brecha en salud que genera la inequidad y la pobreza en zonas rurales -donde justamente el SNS ha mostrado mayor debilidad (24-26) probablemente han contribuido a concentrar los decesos por MC en la población económicamente más débil que, además, enfrenta barreras organizacionales que le impiden el acceso a la atención adecuada, especialmente en situaciones de urgencia (27-30).

El presente trabajo tiene limitaciones. Los estudios de mortalidad en México se basan en los registros oficiales de muertes, los certificados de defunción y las grandes bases de datos gubernamentales, que pueden contener errores en el diagnóstico médico, el llenado de las cédulas y la clasificación de la causa de muerte (31). Además, el subregistro de los nacimientos y las muertes de infantes en las áreas marginadas del país - cuya magnitud varía considerablemente entre regiones - puede aumentar el sesgo del análisis (32). No obstante, estos resultados mantienen su validez, ya que los errores comentados

1. Stemerospects for higher infant survival. World Health Forum. 1990;11(1):78-84.

2. Centers for Disease Control and Prevention. Trends in infant mortality attributable to birth defects-United States, 1980-1995. MMWR. 1998;47:773-8.

3. Hobbs CA, Cleves MA, Simmons CJ. Genetic epidemiology and congenital malformations. Arch Pediatr Adolesc Med. 2002;156(4): 315-20.

4. Chervenak FA, McCullough LB, Skupski D, Chasen ST. Ethical issues in the management of afectan de manera uniforme a todas las MC estudiadas. Por ello, las cifras expuestas constituyen una estimación conservadora del problema.

Como conclusión se puede afirmar que los resultados de este estudio indican que el desarrollo del SNS de México entre 1980 y 2005 no se ha traducido en una reducción en la mortalidad por MC. El reto de las MC se ha enfrentado de una manera parcial, insuficiente e inequitativa, especialmente en los casos con afecciones más graves o que requieren tratamientos de mayor complejidad. Si bien puede que se haya logrado mejorar la eficacia en el diagnóstico de las $\mathrm{MC}$, estos resultados pueden estar reflejando un rezago en la oferta de tratamientos oportunos y la existencia de barreras que impiden la atención de estos pacientes en las unidades de mayor especialización. Ese desequilibrio es estructural y se refleja en la tendencia ascendente en las TMIe por MC graves. Es probable que las mayores afectaciones recaigan en la población económicamente más desfavorecida (33).

Uno de los retos para el SNS mexicano en el siglo XXI es reducir más aún la TMI, para lo que se requiere disminuir la letalidad de las MC. Una recomendación obvia es extender la lucha contra las enfermedades congénitas al terreno de lo prenatal. En lo asistencial, esto implica aprovechar las ventajas que ofrecen las diversas técnicas de diagnóstico prenatal para que los niños con MC nazcan en las unidades que cuentan con el personal y el equipamiento adecuados para su adecuada atención.

En el campo político, se debe respaldar la potestad de la mujer embara- zada para terminar la gestación cuando el embrión es portador de una MC muy grave. Esta pauta, surgida del principio ético de autonomía de la mujer más que de estrategias de salud pública, ha permitido reducir en algunos países las TMI, las muertes fetales tardías y la incidencia y mortalidad por MC (34). Su justificación es clara: contrarrestar la adversidad que enfrentan los padres al traer al mundo a un ser con malformaciones graves, para quien el SNS no tiene las estrategias de acción ni el equipo terapéutico indispensables, según se ha podido comprobar aquí. Lamentablemente, la contaminación de este debate con argumentos propios de otras polémicas hace más difícil en países como México llegar a un consenso al respecto en el futuro próximo. Sin embargo, no por ello la urgencia es menor. Disminuir la TMI será imposible si no se reduce la incidencia de las MC graves y se mejoran las condiciones de atención para estos infantes.

Los sistemas de salud y la sociedad de los diversos países deben asumir un compromiso impostergable con la infancia. El problema generado por las MC solo tendrá solución si se mejoran -cuantitativa y cualitativamente- el diagnóstico prenatal y la atención perinatal de los casos de MC graves, incluida la despenalización de la terminación de los embarazos cuando los embriones presentan MC graves. Se debe prestar especial atención a elevar la cobertura de los servicios mencionados en las poblaciones económicamente menos favorecidas, por ser la principal beneficiaria, y crear un escenario que debe llevar a una mayor equidad.

\section{REFERENCIAS}

pregnancies complicated by foetal anomalies. Obstet Gynecol Surv. 2003;58(7): 473-83.

5. Lara A, Gómez-Dantés O, Urdapilleta O, Bravo ML. Gasto federal en salud en población no asegurada: México 1980-1995. Salud Publica Mex. 1997;39(2):102-9.

6. World Health Organization. World Health Report 2006. Working together for health. Geneva: WHO; 2006.

7. Organización Mundial de la Salud. Manual de la clasificación estadística internacional de enfermedades, traumatismos y causas de defunción. 9. ${ }^{a}$ revisión. Washington, D.C.: OPS; 1975.

8. Organización Mundial de la Salud. Clasificación estadística internacional de enfermedades y problemas relacionados con la salud. 10. a revisión. Lista tabular. Washington, D.C.: OPS; 1995.

9. Consejo Nacional de Población. Indicadores demográficos básicos 1990-2030. México en Cifras. México, D.F.: CONAPO; 2005. Hallado 
en http://www.conapo.gob.mx/00cifras/00 indicadores.htm. Acceso el 12 de mayo de 2007.

10. Reidpath DD, Allotey P. Infant mortality rate as an indicator of population health. J Epidemiol Comm Health. 2003;57(5):344-6.

11. Rosano A, Botto LD, Botting B, Mastroiacovo P. Infant mortality and congenital anomalies from 1950 to 1994: an international perspective. J Epidemiol Comm Health. 2000;54(9): 660-6.

12. Jung SC, Kim SS, Yoon KS, Lee JS. Prevalence of congenital malformations and genetic diseases in Korea. J Hum Genet. 1999;44(1):30-4.

13. MacMahon B. The continuing enigma of pyloric stenosis of infancy: a review. Epidemiology. 2006;17(2):195-201.

14. Terai M, Niwa K, Nakazawa M, Tatsuno K, Segami K, Hamada $\mathrm{H}$, et al. Mortality from congenital cardiovascular malformations in Japan, 1968 through 1997. Circulation J. 2002; 66(5):484-8.

15. Boneva RS, Botto LD, Moore CA, Yang Q, Correa A, Erickson JD. Mortality associated with congenital heart defects in the United States. Trends and racial disparities, 19791997. Circulation. 2001;103(19):2376-81.

16. Yang W, Carmichael SL, Harris JA, Shaw GM. Epidemiologic characteristics of congenital diaphragmatic hernia among 2.5 million California births, 1989-1997. Birth Defects Res A Clin Mol Teratol. 2006;70(10):820-4.

17. De Galan-Roosen AE, Kuijpers JC, Meershoek $\mathrm{AP}$, van Velzen D. Contribution of congenital malformations to perinatal mortality. A 10 years prospective regional study in The Netherlands. Eur J Obstet Gynecol Reprod Biol. 1998;80(1):55-61.

18. Wen SW, Liu S, Joseph KS, Rouleau J, Allen A. Patterns of infant mortality caused by major congenital anomalies. Teratology. 2000;61(5): 342-6.

19. Forrester MB, Merz RD. First-year mortality rates for selected birth defects, Hawaii, 19861999. Am J Med Genet A. 2003;119(3):311-8.

20. Bedregal P, Orfali C, Norero E, Vercellino M. Mortalidad por malformaciones congénitas en Chile (1969-1997). Rev Med Chil. 2000; 128(4):392-8.

21. Ramírez-Espitia JA, Benavides FG, LacasañaNavarro M, Martínez JM, García AM, Benach J. Mortalidad por defectos del tubo neural en México, 1980-1997. Salud Publica Mex. 2003; 45(5):356-64

22. Bhutta ZA, Darmstadt GL, Hasan BH, Haws RA. Community-based interventions for improving perinatal and neonatal health outcomes in developing countries: a review of the evidence. Pediatrics. 2005;115(supl 2): 519-617.

23. Gutiérrez JP, Bertozzi SM. La brecha en salud en México medida a través de la mortalidad infantil. Salud Publica Mex. 2003;45(2): 102-9.

24. Miranda-Ocampo R, Salvatierra-Izaba B, Vivanco-Cedeño B, Álvarez-Lucas C, LezanaFernández MA. Inequidad de los servicios de salud a población abierta en México. Salud Publica Mex. 1993;35(6):576-84.

25. Hernández-Ávila JE, Rodríguez MH, Rodríguez NE, Santos R, Morales E, Cruz C, et al. Cobertura geográfica del sistema mexicano de salud y análisis espacial de la utilización de hospitales generales de la Secretaría de Salud en 1998. Salud Publica Mex. 2002;44(6): 519-32.

26. World Health Organization. The World Health Report 2000. Health systems: improving performance. Geneva: WHO; 2000.
27. Macinko JA, Shi L, Starfield B. Wage inequality, the health system, and infant mortality in wealthy industrialized countries, 1970-1996. Social Sci Med. 2004;58(2):279-92.

28. López-Ríos O. Efecto de los servicios de salud y de factores socioeconómicos en las diferencias espaciales de la mortalidad mexicana. Salud Publica Mex. 1997;39(1):16-24.

29. Bol KA, Rickard RS, Kirby RS. Case fatality among infants with congenital malformations by lethality. Birth Defects Res A Clin Mol Teratol. 2004;70(9):580-5.

30. Cornel MC. Wealth and health in relation to birth defects mortality. J Epidemiol Comm Health. 2000;54(9):644.

31. Maudsley G, Williams EM. Inaccuracy in death certification-where are we now? J Public Health Med. 1996;18(1):59-66.

32. Tomé $P$, Reyes $H$, Piña $C$, Rodríguez L, Gutiérrez G. Características asociadas al subregistro de muerte en niños del estado de Guerrero, México. Salud Publica Mex. 1997;39(6): 523-9.

33. Aguilera N, Marrufo GM. Can better infrastructure and quality reduce hospital infant mortality rates in Mexico? Health Policy. 2007;80(2) 239-52.

34. Liu S, Joseph KS, Kramer MS, Allen AC, Sauve R, Rusen ID, et al. Relationship of prenatal diagnosis and pregnancy termination to overall infant mortality in Canada. J Am Med Assoc. 2002;287(12):1561-7.

Manuscrito recibido el 5 de julio de 2007. Aceptado para publicación, tras revisión, el 27 de mayo de 2008.

ABSTRACT Objectives. To evaluate the role that Mexico's National Health System (Sistema Nacional de Salud-SNS) has played in the task of reducing the number of deaths due to congenital malformations through a trends analysis of cause-specific infant mortality rates (IMRcs).

Methods. Time-series analysis of deaths of boys and girls under 1 year of age from 1980-2005, according to databases of national and annual mortality maintained by the Secretariat of Health of Mexico. Cause-specific mortality rates were calculated for the most frequently occurring, severe, congenital malformations in Mexico: neural tube defects, diaphragmatic hernias, exomphalos (omphalocele and gastroschisis), and heart and digestive tract defects, grouped according to severity, degree of technological sophistication required for treatment, and most frequent outcome.

Results. From 1980-2005, the infant mortality rate in Mexico decreased from 40.7 to 16.9 per 1000 births $(\beta=-0.86 ; P<0.001)$; however, the mortality rate for congenital malformations rose from 2.2 to 3.5 per 1000 births $(\beta=0.05 ; P<0.001)$. Only infantile hypertrophic pyloric stenosis and anorectal atresia, anomalies with good prognoses and treatments available in minimally-equipped facilities, exhibited downward trends in their IMRcs $(\beta=-0.01$ to $-0.09 ; P<0.001)$; while malformations requiring immediate treatment in specialized facilities showed rising IMRcs $(\beta=0.03$ to $0.05 ; P<0.001)$. Conclusions. The development of Mexico's SNS from 1980-2005 has not translated into a reduction of mortality from congenital malformations; this deficiency was more pronounced for anomalies that require immediate treatment and sophisticated technology.

Key words Infant mortality, infant mortality rate, congenital abnormalities, Mexico. 